\title{
Transformation Algorithm of Dielectric Response in Time-Frequency Domain
}

\author{
Ji Liu, ${ }^{1}$ Daning Zhang, ${ }^{1}$ Xinlao Wei, ${ }^{1}$ and Hamid Reza Karimi ${ }^{2}$ \\ ${ }^{1}$ State Key Laboratory Breeding Base of Dielectrics Engineering, Harbin University of Science and Technology, \\ Harbin, Heilongjiang 150080, China \\ ${ }^{2}$ Department of Engineering, Faculty of Engineering and Science, University of Agder, 4898 Grimstad, Norway
}

Correspondence should be addressed to Ji Liu; liuji@hrbust.edu.cn

Received 21 March 2014; Revised 23 April 2014; Accepted 25 April 2014; Published 1 June 2014

Academic Editor: Kwok-Wo Wong

Copyright (C) 2014 Ji Liu et al. This is an open access article distributed under the Creative Commons Attribution License, which permits unrestricted use, distribution, and reproduction in any medium, provided the original work is properly cited.

\begin{abstract}
A transformation algorithm of dielectric response from time domain to frequency domain is presented. In order to shorten measuring time of low or ultralow frequency dielectric response characteristics, the transformation algorithm is used in this paper to transform the time domain relaxation current to frequency domain current for calculating the low frequency dielectric dissipation factor. In addition, it is shown from comparing the calculation results with actual test data that there is a coincidence for both results over a wide range of low frequencies. Meanwhile, the time domain test data of depolarization currents in dry and moist pressboards are converted into frequency domain results on the basis of the transformation. The frequency domain curves of complex capacitance and dielectric dissipation factor at the low frequency range are obtained. Test results of polarization and depolarization current (PDC) in pressboards are also given at the different voltage and polarization time. It is demonstrated from the experimental results that polarization and depolarization current are affected significantly by moisture contents of the test pressboards, and the transformation algorithm is effective in ultralow frequency of $10^{-3} \mathrm{~Hz}$. Data analysis and interpretation of the test results conclude that analysis of time-frequency domain dielectric response can be used for assessing insulation system in power transformer.
\end{abstract}

\section{Introduction}

Most of dangerous breakdowns in high voltage (HV) apparatus are caused by the aging effects of $\mathrm{HV}$ insulation system. Reliable diagnostics for insulation system are correspondingly based on changes of the dielectric properties. Generally, insulation diagnosis methods for large oilimpregnated power transformer include frequency domain method (frequency domain spectroscopy, FDS) [1], time domain voltage method (return voltage meter, RVM) [2], and current method (polarization and depolarization current, PDC) [3]. RVM can only be used for analyzing depolarization process of dielectric. Nevertheless, the change of dielectric conductivity cannot be shown in RVM. Many researchers focus their attention on the analysis of time domain and frequency domain dielectric responses which has been used for diagnosing insulation aging of high voltage apparatus $[4,5]$. As for FDS, dielectric dissipation factor $\tan \delta(\omega)$ and dielectric capacitance $C(\omega)$ are investigated at the range of low and ultralow frequency, which can represent relaxation characteristics, such as interfacial polarization and space charge polarization widely found in most of the dielectrics. With regard to PDC, comparing the PDC curves between new factory oil and aged transformer oil indicates the operating conditions of on-site power transformers [6].

The presentation of dielectric response in frequency domain has advantages. The real and imaginary part of the complex capacitance can be separated and the dissipation factor is defined in frequency domain. Transformation of time domain to frequency domain is helpful to interpret data better $[7,8]$. Compared with FDS, fast on-site measurement to dielectric characteristics can be accomplished by the use of PDC test. Unlike the limited accuracy to PDC in higher frequency range, FDS analysis can be utilized over a wide range of frequency. In traditional FDS, nevertheless, we need a long time at very low frequency measurement. In 
this paper, the principles of dielectric response are analyzed theoretically on the basis of the analysis of polarization and depolarization current, and frequency domain properties of dielectric dissipation factor will be obtained according to transform algorithm of depolarization current data, which provide a convenient and fast measurement for the low frequency characteristics of dielectric dissipation factor.

\section{Transform Algorithm}

2.1. Dielectric Response in Time Domain. Assuming a homogeneous electric field $E(t)$ is applied over a vacuum-insulated electrode arrangement, the electric displacement $D(t)$ is proportional to the electric field $E(t)$;

$$
D(t)=\varepsilon_{0} E(t),
$$

where $\varepsilon_{0}$ is vacuum permittivity.

If any kind of isotropic dielectric materials replaces the vacuum, the electric displacement $D(t)$ will grow as a result of additional electrical (macroscopic) polarization $P(t)$, and (1) can be rewritten as

$$
D(t)=\varepsilon_{0} E(t)+P(t) .
$$

The electrical polarization $P(t)$ can be divided into two parts: one represents "rapid" polarization processes and the other represents "slow" polarization processes. The electrical polarization can be written as

$$
P(t)=\varepsilon_{0}\left(\varepsilon_{\infty}-1\right) E(t)+\varepsilon_{0} \int_{-\infty}^{t} f(t-\tau) E(\tau) d \tau,
$$

where $\varepsilon_{\infty}$ is optical frequency permittivity. The "rapid" polarization follows the applied electric field, whereas "slow" polarization is built up from a convolution integral between the applied electric field and a function which is called the dielectric response function $f(t)$. The dielectric response function represents the "memory" effects in a dielectric material and has the following characteristics:

$$
f(t) \equiv 0, \quad \forall t<0 .
$$

In accordance with Ampere's law $[9,10]$, the electric field $E(t)$ generates a total current density $J(t)$, which can be written as a sum of conduction, vacuum, and polarization displacement current:

$$
\begin{aligned}
J(t)= & \sigma_{0} E(t)+\varepsilon_{0} \varepsilon_{\infty} \frac{d E(t)}{d t} \\
& +\varepsilon_{0} \frac{d}{d t} \int_{0}^{t} f(t-\tau) E(\tau) d \tau .
\end{aligned}
$$

According to the theory of dielectric response, when fixed electric field $E(t)$ generated by an external voltage $U(t)$ is applied to isotropic dielectric material, the total current $i(t)$ in dielectric can be written as

$$
i(t)=C_{0}\left[\frac{\sigma_{0}}{\varepsilon_{0}} U(t)+\varepsilon_{\infty} \frac{d U(t)}{d t}+\frac{d}{d t} \int_{0}^{t} f(t-\tau) U(\tau) d \tau\right],
$$

where $\sigma_{0}$ is dielectric volume conductivity, applied voltage $U(t)=E(t) \cdot d, C_{0}$ is geometry capacitance between electrodes, and $d$ is the spacing between the two electrodes.

For characterizing dielectric response in time domain, a step dc "charging voltage" with magnitude $U_{C}$, which must be constant and free of ripple, is suddenly applied to the test sample which has been discharged previously. Then the polarization current $i_{\text {pol }}(t)$ flowing through the dielectric can be recorded as

$$
i_{\mathrm{pol}}(t)=C_{0} U_{C}\left[\frac{\sigma_{0}}{\varepsilon_{0}}+\varepsilon_{\infty} \delta(t)+f(t)\right],
$$

where $\delta(t)$ is the delta function arising from the suddenly applied step voltage at $t=0$. The charging current $i_{\text {pol }}(t)$ contains three parts: the first one is related to the inherent conductivity of the test object and is independent of any polarization process, the middle part with the delta function cannot be recorded in practice and is always ignored in the calculation due to the large dynamic range of current amplitudes arising from the very fast polarization processes at first, and the last one represents all the "slow" polarization processes during the applied voltage. Therefore, (7) can be rewritten as

$$
i_{\text {pol }}(t)=C_{0} U_{C}\left[\frac{\sigma_{0}}{\varepsilon_{0}}+f(t)\right] .
$$

If the step voltage $U_{C}$ is removed and the testing dielectric is short-circuited when $t=t_{c}$, the depolarization current $i_{\text {depol }}$ can be measured. As shown in Figure $1, T_{c}$ is the time duration when the step voltage was applied. Figure 1 shows the changing curve of polarization and depolarization current. According to the superposition principle, the sudden decrease of $U_{C}$ can be regarded as a negative step voltage $-U_{C}$ applied at time $t=t_{c}$; we get the depolarization current $i_{\text {depol }}$ :

$$
i_{\text {depol }}(t)=-C_{0} U_{C}\left[f(t)-f\left(t+t_{c}\right)\right] .
$$

The second part in (9) can be neglected if the charging time $t_{c}$ is long enough to complete all the polarization processes. Then, the depolarization current becomes proportional to the dielectric response function $f(t)$ :

$$
f(t) \approx \frac{i_{\mathrm{depol}}}{C_{0} U_{C}} .
$$

If the test period is long enough, the conductivity $\sigma_{0}$ can easily be calculated from the polarization and depolarization currents:

$$
\sigma_{0} \approx \frac{\varepsilon_{0}}{C_{0} U_{C}}\left[i_{\text {pol }}(t)+i_{\text {depol }}(t)\right]
$$

2.2. Dielectric Response in Frequency Domain. Assuming that voltage $U(\omega)$ is given, frequency domain current will be obtained by applying the Fourier transform to total current $i(t)$. Consider

$$
\begin{aligned}
I(\omega) & =C_{0}\left[\frac{\sigma_{0}}{\varepsilon_{0}} U(\omega)+j \omega \varepsilon_{\infty} U(\omega)+j \omega F(\omega) U(\omega)\right] \\
& =\left[\frac{\sigma_{0}}{\varepsilon_{0}}+j \omega\left(\varepsilon_{\infty}+F(\omega)\right)\right] C_{0} U(\omega) .
\end{aligned}
$$




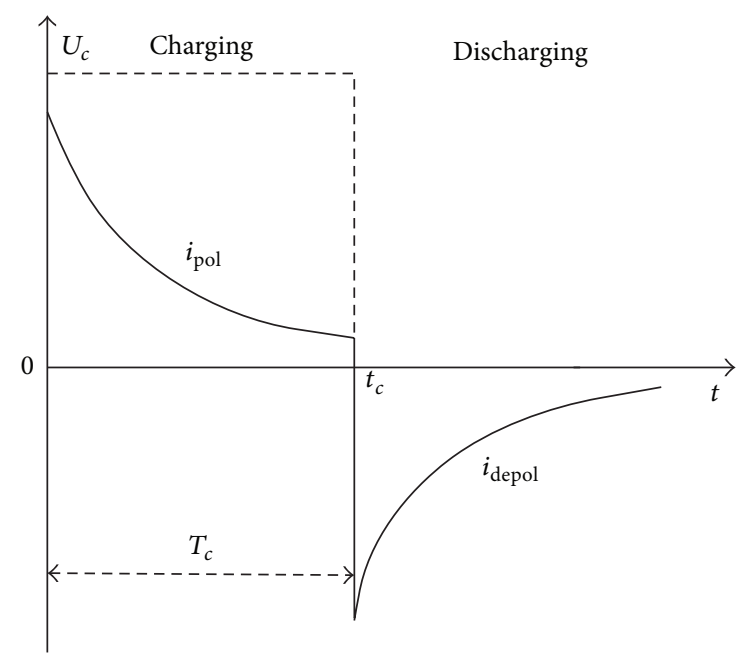

FIGURE 1: Polarization and depolarization current.

Obviously, $F(\omega)$ is Fourier transform value of dielectric response function $f(t)$, namely, complex dielectric susceptibility. The Fourier transform is the link between time and frequency domain. The principle of superposition is effective if the dielectric material is linear, homogenous, and isotropic. Formula (13) shows the relationship between time and frequency domain. Consider

$$
\chi(\omega)=F(\omega)=\chi^{\prime}(\omega)-j \chi^{\prime \prime}(\omega)=\int_{0}^{\infty} f(t) e^{-j \omega t} d t .
$$

According to (12), we have

$$
\begin{aligned}
I(\omega) & =\left[\frac{\sigma_{0}}{\varepsilon_{0}}+j \omega\left(\varepsilon_{\infty}+\chi^{\prime}(\omega)-j \chi^{\prime \prime}(\omega)\right)\right] C_{0} U(\omega) \\
& =\left[\frac{\sigma_{0}}{\varepsilon_{0}}+\omega \chi^{\prime \prime}(\omega)+j \omega\left(\varepsilon_{\infty}+\chi^{\prime}(\omega)\right)\right] C_{0} U(\omega) .
\end{aligned}
$$

Therefore, frequency domain expression of total current in dielectric material under $U(\omega)$ is

$$
\begin{aligned}
I(\omega) & =j \omega C_{0}\left[\varepsilon_{\infty}+\chi^{\prime}(\omega)-j\left(\frac{\sigma_{0}}{\varepsilon_{0} \omega}+\chi^{\prime \prime}(\omega)\right)\right] U(\omega) \\
& =j \omega C_{0} \dot{\varepsilon}(\omega) U(\omega),
\end{aligned}
$$

where complex permittivity is

$$
\dot{\varepsilon}(\omega)=\varepsilon^{\prime}(\omega)-j \varepsilon^{\prime \prime}(\omega) .
$$

In many cases, it is more convenient to use the complex permittivity instead of the complex dielectric susceptibility. The dielectric dissipation factor in frequency domain can therefore be defined as follows:

$$
\tan \delta(\omega)=\frac{\varepsilon^{\prime \prime}(\omega)}{\varepsilon^{\prime}(\omega)}=\frac{\left(\sigma_{0} / \varepsilon_{0} \omega\right)+\chi^{\prime \prime}(\omega)}{\varepsilon_{\infty}+\chi^{\prime}(\omega)} .
$$

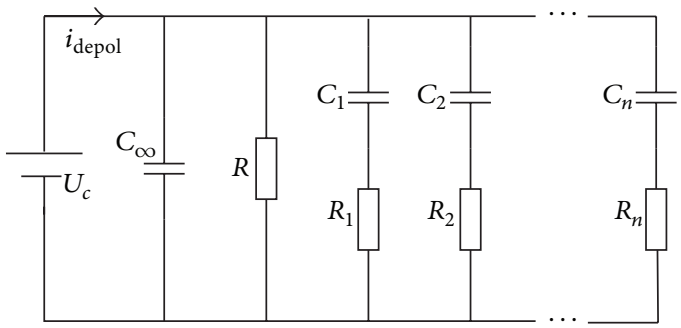

FIGURE 2: Equivalent circuit of a linear dielectric.

2.3. Transformation of Dielectric Response. As mentioned before, the Fourier transform is the link between time and frequency domain $[11,12]$. If the dielectric response function $f(t)$ follows the "Curie-von Schweidler" model, its Fourier transform can be calculated as follows:

$$
\begin{aligned}
\chi(\omega) & =F(\omega)=\int_{0}^{\infty} A t^{-n} e^{-j \omega t} d t=\frac{A \cdot \Gamma(1-n)}{(j \omega)^{1-n}} \\
& =A \cdot \Gamma(1-n) \cdot \omega^{n-1}\left(\sin \left(\frac{n \pi}{2}\right)-j \cos \left(\frac{n \pi}{2}\right)\right),
\end{aligned}
$$

where $\Gamma$ is the Gamma function. Many results confirm that this method is effective with a narrow frequency range [13].

If the measured depolarization current can be approximated by a piecewise "Curie-von Schweidler" model, Hamon approximation [14] is an alternative for fast calculation of the time domain data into frequency domain. But only the imaginary part of the complex permittivity $\varepsilon^{\prime \prime}$ can be calculated by the method. If $n$ is in the range of $0.3<n<1.2$, the imaginary part of the complex permittivity can be written as

$$
\varepsilon^{\prime \prime}(\omega) \approx \frac{-i_{\mathrm{depol}}(0.1 / f)}{2 \pi f C_{0} U_{c}}
$$

It is shown that the imaginary part of the complex permittivity at a frequency of $f$ cycles per second can be obtained directly from the current at a time $t$ equal to $0.1 / f$ seconds after applying a direct step voltage. Since the depolarization current is easily measured if $t$ is large enough, the method is adapted to the rapid evaluation of loss factor at frequency below 0.01 cycles per second, where direct measurement would be tedious or time consuming.

According to dielectric Debye model [15], as shown in Figure 2, the depolarization current of linear insulation system can be expressed by the superposition of different relaxation current components. When charging time is long enough, dielectric response function $f(t)$ is proportional to polarization current. Consider

$$
f(t)=\frac{1}{C_{0} U_{c}} \sum_{i=1}^{n} A_{i} e^{-t / \tau_{i}}
$$

The complex susceptibility can be obtained as

$$
\chi(\omega)=\int_{0}^{\infty} f(t) e^{-j \omega t} d t=\frac{1}{C_{0} U_{c}} \sum_{i=1}^{n} \frac{A_{i} \tau_{i}-j A_{i} \omega \tau_{i}^{2}}{1+\left(\omega \tau_{i}\right)^{2}} .
$$


Thus, real part and imaginary part of susceptibility can be obtained in (21) by

$$
\begin{aligned}
\chi^{\prime}(\omega) & =\frac{1}{C_{0} U_{c}} \sum_{i=1}^{n} \frac{A_{i} \tau_{i}}{1+\left(\omega \tau_{i}\right)^{2}}, \\
\chi^{\prime \prime}(\omega) & =\frac{1}{C_{0} U_{c}} \sum_{i=1}^{n} \frac{A_{i} \omega \tau_{i}^{2}}{1+\left(\omega \tau_{i}\right)^{2}} .
\end{aligned}
$$

Therefore, the dielectric dissipation factor in (17) can be rewritten as

$$
\begin{aligned}
\tan \delta(\omega) & =\frac{\varepsilon^{\prime \prime}(\omega)}{\varepsilon^{\prime}(\omega)}=\frac{\left(\sigma_{0} / \varepsilon_{0} \omega\right)+\chi^{\prime \prime}(\omega)}{\varepsilon_{\infty}+\chi^{\prime}(\omega)} \\
& =\frac{\left(\sigma_{0} / \varepsilon_{0} \omega\right)+\left(1 / C_{0} U_{c}\right) \sum_{i=1}^{n}\left(A_{i} \omega \tau_{i}^{2} /\left(1+\left(\omega \tau_{i}\right)^{2}\right)\right)}{\varepsilon_{\infty}+\left(1 / C_{0} U_{c}\right) \sum_{i=1}^{n}\left(A_{i} \tau_{i} /\left(1+\left(\omega \tau_{i}\right)^{2}\right)\right)} .
\end{aligned}
$$

\section{Test System}

The test samples are pressboards with the thickness of $1 \mathrm{~mm}$. After polarization and depolarization current measurement, the frequency domain dielectric response is obtained through the above transformation; thus, dielectric dissipation factor curves in low frequency can be illustrated. The experimental system mainly consists of high-voltage dc power supply, Keithley 6517B electrostatic meter, testing electrode box, control switch, and PC. The schematic diagram of experimental circuit is shown in Figure 3. A dc high voltage can be changed continuously with maximum output voltage of $5 \mathrm{kV}$. The three-electrode system is used with $50 \mathrm{~mm}$ diameter of measure electrode and $2 \mathrm{~mm}$ protection gap.

\section{Results and Discussions}

For verifying the transform of dielectric response from time to frequency domain, the pressboard samples with thickness of $1 \mathrm{~mm}$ were used. Applied polarization voltages, respectively, were $250 \mathrm{~V}, 500 \mathrm{~V}$, and $1000 \mathrm{~V}$ with polarization time of $100 \mathrm{~s}$. For comparing conveniently, the polarization current was given in the absolute value. Experimental results are shown in Figure 4, which prove that the amplitude of polarization and depolarization current will be affected by different polarization voltages. Moreover, there are the same declined trends to the curves of polarization and depolarization current individually. The higher the polarization voltage is, the bigger the current amplitude is. It is shown from test results that there are better linear characteristics for the pressboards.

Because of the long polarization time for insulation material, the process of dielectric polarization is more sufficient and there is more information related to polarized pattern, which is more suitable for insulation condition assessment of the insulation system. The phenomena can be called hereby "memory effect." In order to explain it, the experiments were carried out with the pressboard of $1 \mathrm{~mm}$ thickness, polarization voltage of $500 \mathrm{~V}$, and polarization time of $100 \mathrm{~s}$,
$200 \mathrm{~s}$, and $400 \mathrm{~s}$ individually, as shown in Figure 5. The dependence of depolarization current is roughly coincident within initial $30 \mathrm{~s}$, but the curves of depolarization current have upward tendency with the increase of polarization time. The phenomena illustrate adequately that more bounded charge will appear with full polarization process in dielectric, and more internal information can be obtained.

As a strong polar molecule for ageing by-products of pressboard, polarization and depolarization currents are very sensitive to moisture. Two similar pressboards of $1 \mathrm{~mm}$ were, respectively, marked as A and B. Samples A and B were put into drying oven for heating and drying under the same conditions. Then, sample B was put in damp environment maintaining up to 24 hours with absorption of moisture. The polarization and depolarization currents of $\mathrm{A}$ and $\mathrm{B}$ were tested with applied voltage of $500 \mathrm{~V}$, polarization and depolarization time of 2000 s. As shown in Figure 6, current amplitude of sample B becomes higher than that of sample A due to the absorption of moisture. In addition, when the voltage is applied, because of strong polarity of moisture, the more moisture is produced, the more bounded charges appear; therefore, the depolarization process will slow down. According to the above theoretical analysis, discrete signals of depolarization current in dry and moisture pressboard were probed at first. Test data were fitted according to polynomial curve to generate the time domain formula and were calculated by the use of the transform algorithm to get the frequency domain properties of pressboards. The geometric capacitance of model is $17.4 \mathrm{pF}$. The dielectric dissipation factor, real part and imaginary part of complex capacitance in frequency domain are shown in Figures 7-9.

As shown in Figure 7, dielectric dissipation factor of sample $B$ is higher than that of sample $A$, and curves of dielectric dissipation factor decline slowly with increasing frequency. As shown in Figures 8 and 9, the calculated real part of complex capacitance is identical with both discrete and continuous transformations. The complex capacitance of dry pressboard A is not changed obviously in the whole frequency range.

Nevertheless, the real part of complex capacitance increases clearly for the moist pressboard $\mathrm{B}$, and the difference between A and B becomes very little with the increase of frequency. Calculated curves of discrete transform are coincident with value of continuous transform in the former part of the curves. The former is less than the latter part, and there is no excessive error. With regard to the real part of capacitance, the change dependence between pressboards $\mathrm{A}$ and $\mathrm{B}$ was basically identical, and only the amplitude of pressboard B is higher than that of A. Therefore, imaginary part of the complex capacitance will be increased due to existing moisture.

According to the theoretical and experimental results here, presented transformation algorithm and derived formula can not only carry out measurements more rapid in contrast to the PDC test, but also show more changing patterns of dielectric parameters from the response curves. As shown in Figure 8, different effects of moisture on dependence of real part complex capacitance are obvious. The distinctions are more evident at the frequency range 


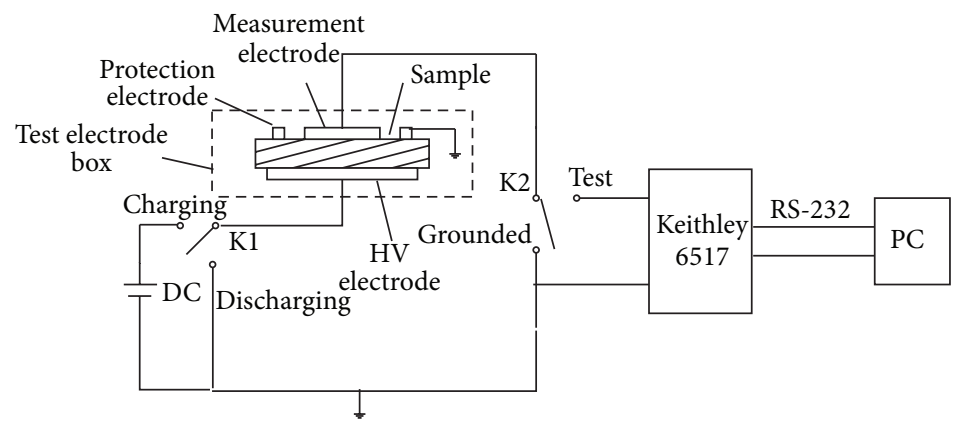

FIgURE 3: Schematic diagram of test system.

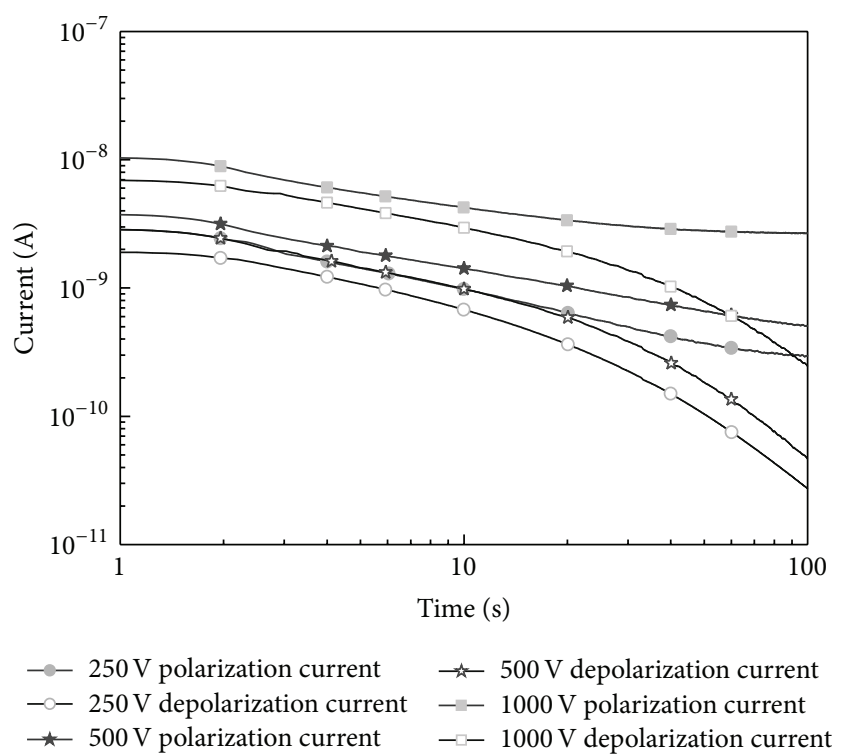

FIGURE 4: Effect of different polarization voltage on PDC.

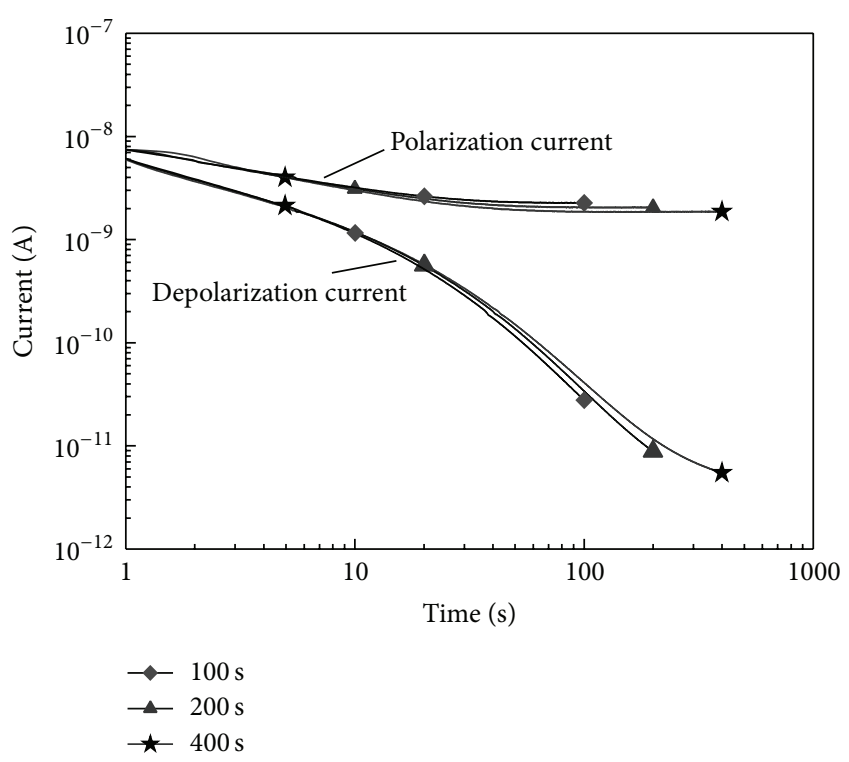

FIGURE 5: Effect of different polarization time on PDC.

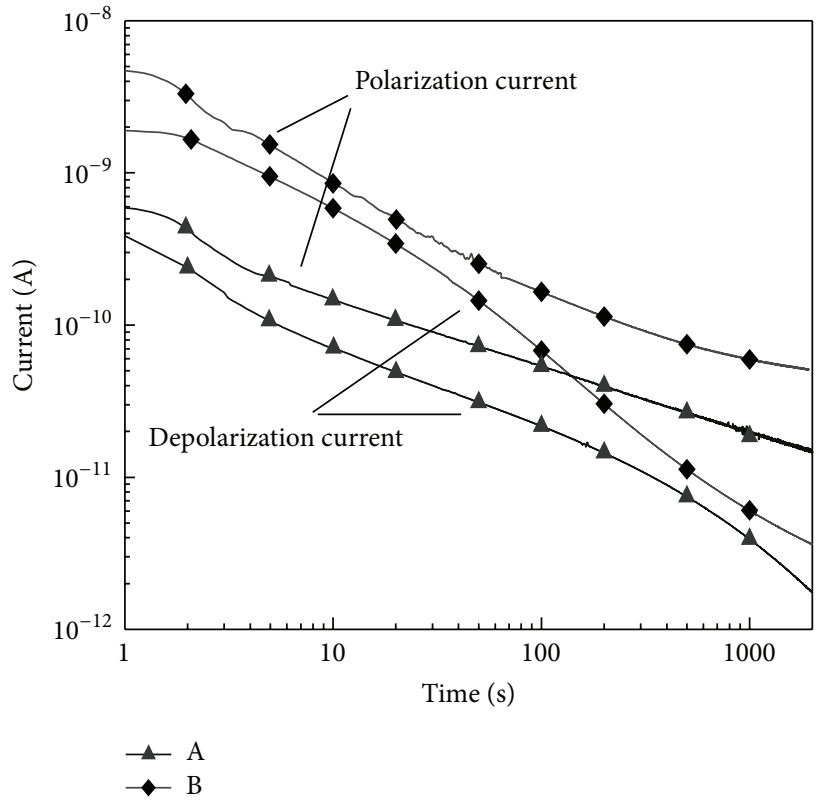

FIGURE 6: Effect of different moisture on PDC.

of lower $10^{-2} \mathrm{~Hz}$. However, for the measurement results of depolarization current in PDC method $[6,13,16]$, the relaxation current cannot be used for distinguishing different dielectric relaxation behavior unlike the FDS curves at certain frequency ranges.

\section{Conclusions}

Based on theoretical analysis in this paper, the test results of polarization and depolarization current in pressboards are given at different voltage and polarization times. It is shown from the experimental results that the polarization and depolarization currents are affected significantly by moisture contents of the pressboard samples. In addition, linear characteristics of pressboard are explained, and it is shown that more sufficient polarization process can help obtain further internal information of dielectric. The dependence of curves of dielectric dissipation factor in frequency domain, which is obtained by calculation, shows that dielectric dissipation 


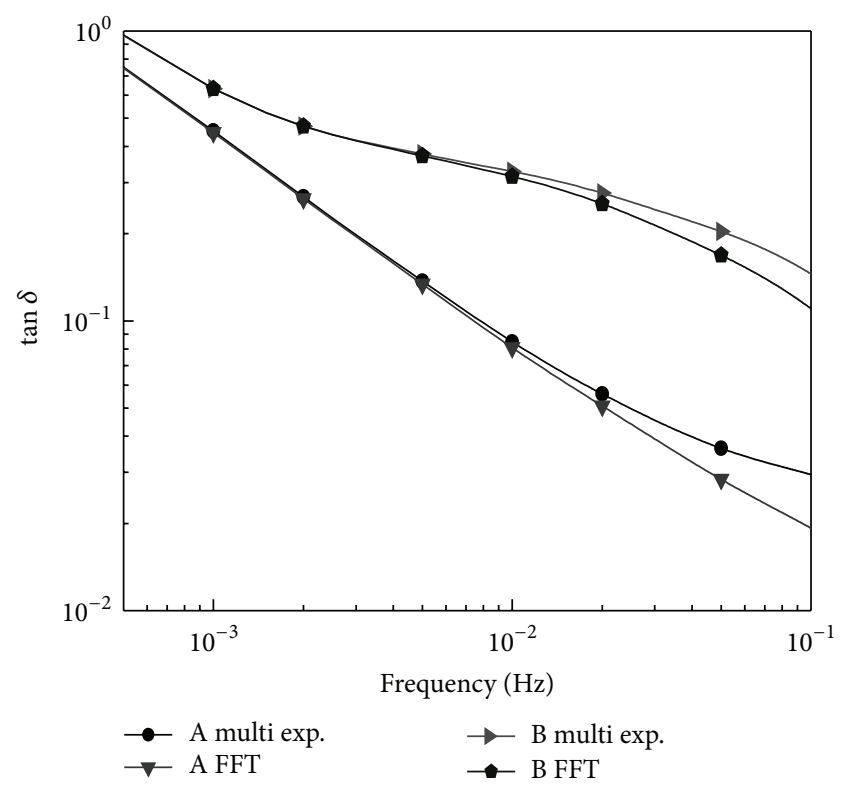

Figure 7: Dependence of dielectric dissipation factor in frequency domain.

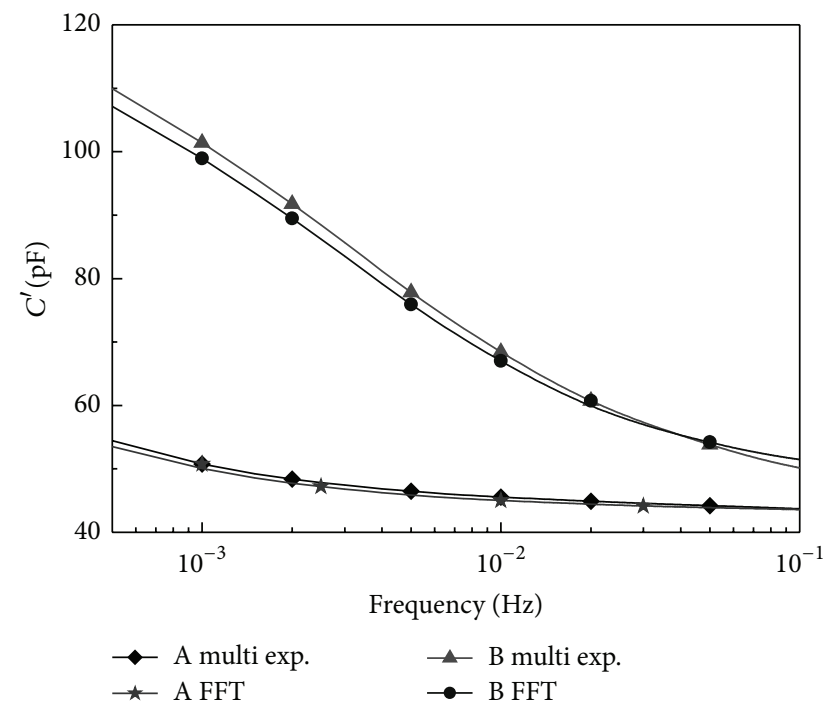

FIGURE 8: Dependence of real part of complex capacitance.

factor will shift upwards with the increase of moisture and be flat with increased frequency.

Comparing the calculating results with actual test data, there is a coincidence for both results in low frequency range, which demonstrates the feasibility and practicability. It is concluded that dielectric dissipation factor in the low frequency range can be calculated by the use of the transform algorithm to the depolarization current in time domain, which can replace test value in low frequency range; consequently, it is a rapid assessment method for dielectric diagnosis.

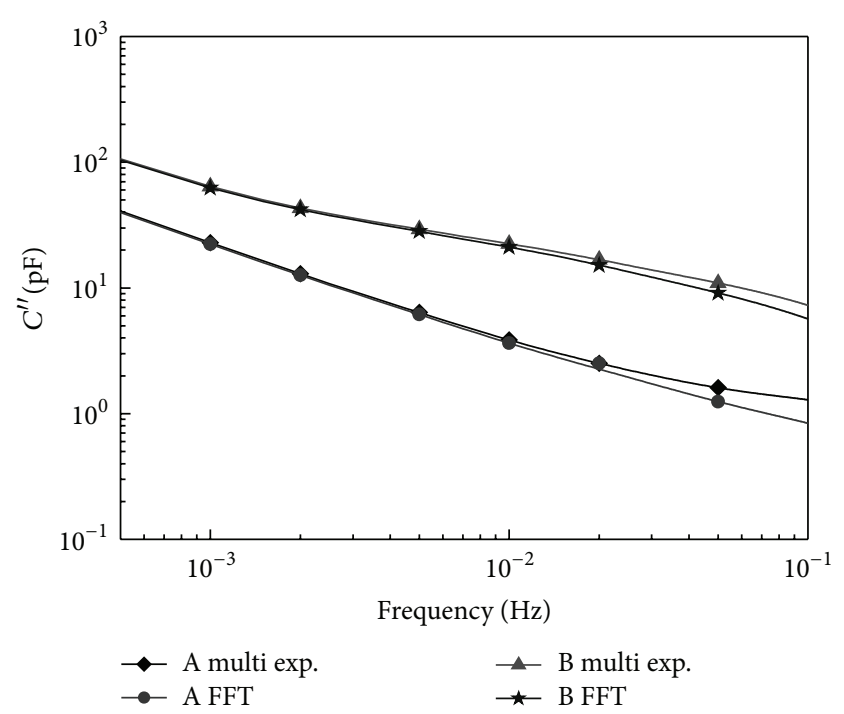

FIGURE 9: Dependence of imaginary part of complex capacitance.

\section{Conflict of Interests}

The authors declare that there is no conflict of interests regarding the publication of this paper.

\section{Acknowledgments}

This project was supported by the National Basic Research Program of China (2012CB723308). The authors wish to thank the Key Laboratory of Engineering Dielectric and Its Application of Ministry of Education, State Key Laboratory Breeding Base of Dielectrics Engineering, for their support.

\section{References}

[1] J. Blennow, C. Ekanayake, K. Walczak, B. García, and S. M. Gubanski, "Field experiences with measurements of dielectric response in frequency domain for power transformer diagnostics," IEEE Transactions on Power Delivery, vol. 21, no. 2, pp. 681$688,2006$.

[2] R. G. Zhang, M. Dong, G. J. Zhang, and Z. Yan, "Investigation of return voltage measurement for the assessment of power transformers," in Proceedings of the International Conference on Condition Monitoring and Diagnosis (CMD '08), pp. 902-905, Beijing, China, April 2008.

[3] T. K. Saha, "Review of time-domain polarization measurements for assessing insulation condition in aged transformers," IEEE Transactions on Power Delivery, vol. 18, no. 4, pp. 1293-1301, 2003.

[4] A. A. Shayegani, H. Borsi, E. Gockenbach, and H. Mohseni, "Transformation of time domain spectroscopy data to frequency domain data for impregnated pressboard," in Proceedings of the Annual Report Conference on Electrical Insulation and Dielectric Phenomena (CEIDP '04), pp. 162-165, Boulder, Colo, USA, October 2004.

[5] M. Farahani, H. Borsi, and E. Gockenbach, "Dielectric response studies on insulating system of high voltage rotating machines," 
IEEE Transactions on Dielectrics and Electrical Insulation, vol. 13, no. 2, pp. 383-393, 2006.

[6] S. A. Bhumiwat and P. Phillips, "Verification of on-site oil reclamation process by means of polarisation / depolarisation current analysis," in Proceedings of the Conference Record of IEEE International Symposium on Electrical Insulation, pp. 105-108, San Juan, Puerto Rico, September 2004.

[7] N. Issarachai, "Dynamic events analysis of Thailand and Malaysia power systems by discrete wavelet decomposition and short term fourier transform based on GPS synchronized phasor data," International Journal of Innovative Computing, Information and Control, vol. 9, no. 5, pp. 2203-2228, 2013.

[8] M. Fallahpour and D. Megias, "High capacity robust audio watermarking scheme based on FFT and linear regression," International Journal of Innovative Computing, Information and Control, vol. 8, no. 4, pp. 2477-2489, 2012.

[9] J. C. Maxwell, A Treatise on Electricity and Magnetism, Clarendon Press, Oxford, UK, 1981.

[10] W. S. Zaengl, "Dielectric spectroscopy in time and frequency domain for HV power equipment, part I: theoretical considerations," IEEE Electrical Insulation Magazine, vol. 19, no. 5, pp. 5-19, 2003.

[11] N. Zakaria, A. J. Pal, and S. N. M. Shah, "Stagewise optimization of distributed clustered finite difference time domain (FDTD) using genetic algorithm," International Journal of Innovative Computing, Information and Control, vol. 9, no. 6, pp. 23032326, 2013.

[12] M. Nachidi, F. Tadeo, and A. Benzouia, "Controller design for Takagi-Sugeno systems in continuous-time," International Journal of Innovative Computing, Information and Control, vol. 8, no. 9, pp. 6389-6400, 2012.

[13] V. Der Houhanessian, Measurement and analysis of dielectric response in oil-paper insulation system [Ph.D. thesis], Swiss Federal Institute of Technology, ETH, Zurich, 1998.

[14] B. V. Hamon, "An approximate method for deducing dielectric loss factor from direct-current measurement," Proceedings of the IEE II: Power Engineering, vol. 99, no. 27, pp. 151-155, 1952.

[15] T. K. Saha, R. Middleton, and A. Thomas, "Understanding frequency \& time domain polarisation methods for the insulation condition assessment of power transformers," in Proceedings of the IEEE Power and Energy Society General Meeting (PES '09), Calgary, Canada, July 2009.

[16] V. Der Houhanessian and W. S. Zaengl, "Time domain measurements of dielectric response in oil-paper insulation systems," in Proceedings of the IEEE International Symposium on Electrical Insulation, pp. 47-52, Montreal, Canada, 1996. 


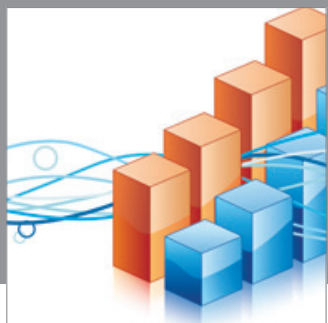

Advances in

Operations Research

mansans

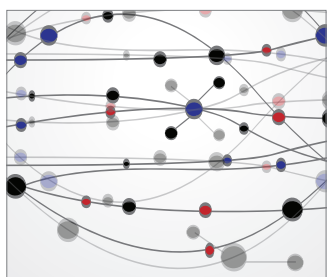

The Scientific World Journal
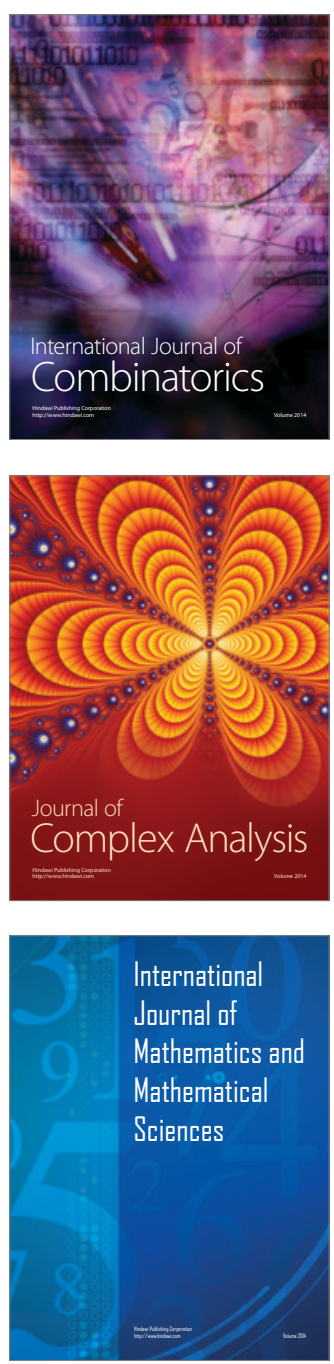
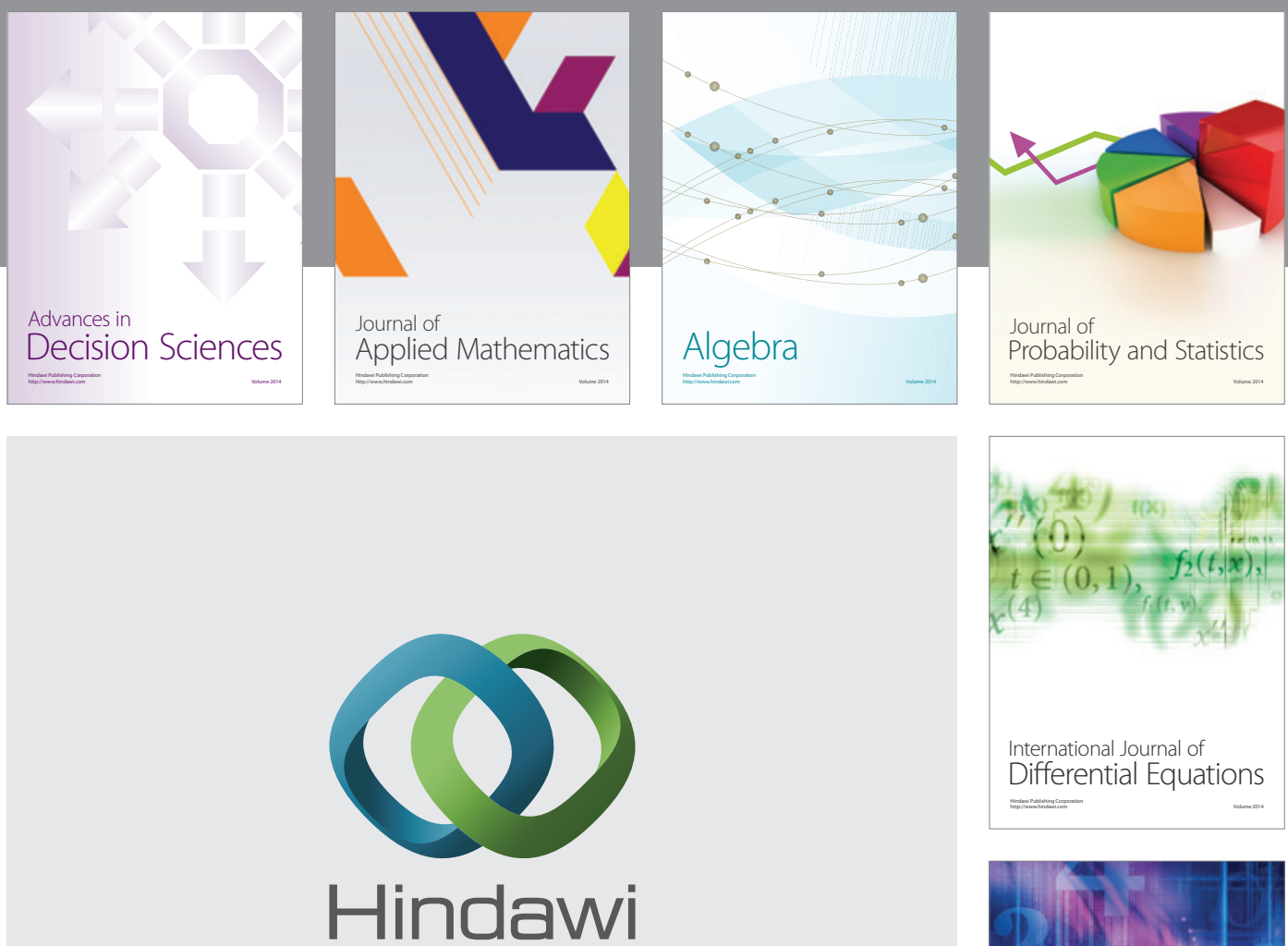

Submit your manuscripts at http://www.hindawi.com
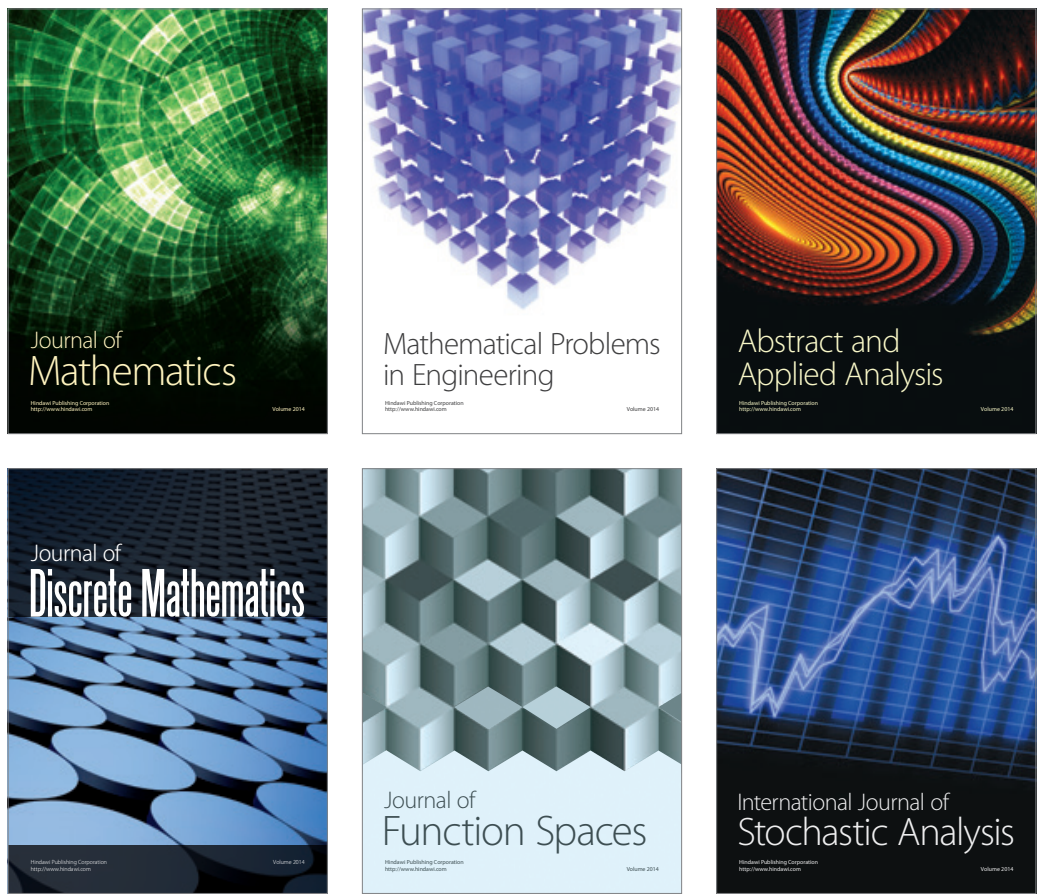

Journal of

Function Spaces

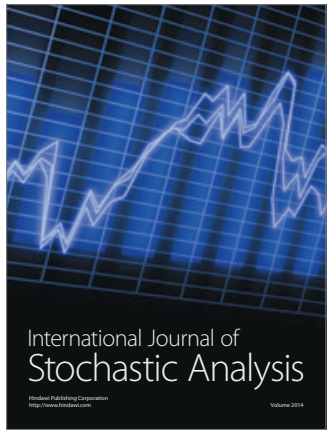

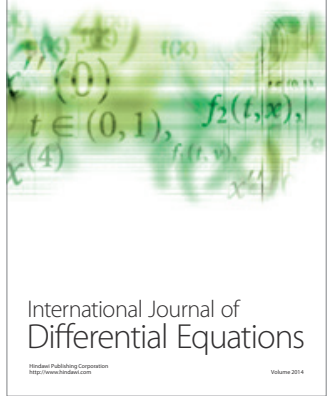
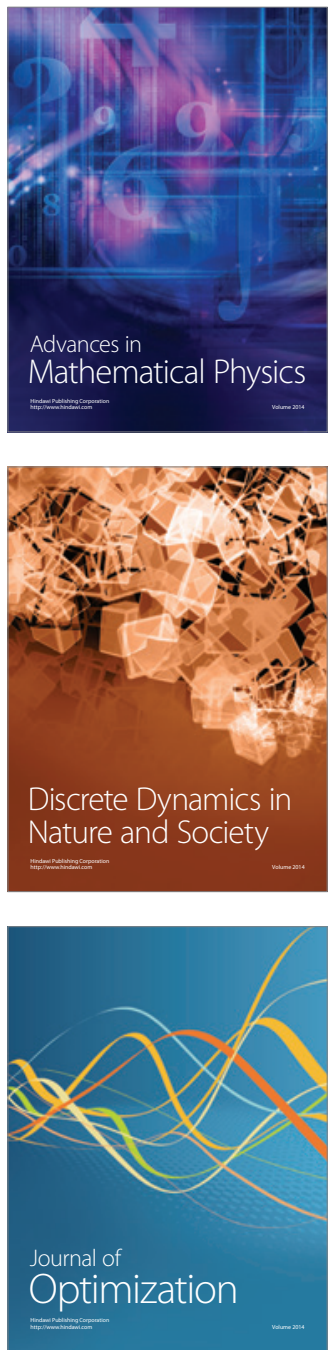